\title{
Duplicity of the Inferior Vena Cava's Image Finding: Clinical and Surgical Care
}

\author{
Beatriz de Andrade Lima Marinho, ${ }^{1}$ Victor Maciel Bandim, ${ }^{1}$ Vivienne Maria Ferreira de Andrade, ${ }^{1}$ Luísa de Andrade Lima \\ Marinho, ${ }^{1}$ Milena Torres Araújo Cavalcanti, ${ }^{1}$ José Guido Corrêa de Araújo Júnior, ${ }^{2}$ Jaciel Benedito de Oliveira ${ }^{3}$ \\ 'Undergraduate Student of Medicine, Medical School, Medical Sciences Center (CCM), Federal University of Pernambuco (UFPE), Recife, PE, \\ Brazil \\ ${ }^{2}$ Professor at the Surgery Department, Medical Sciences Center (CCM), Federal University of Pernambuco (UFPE), Recife, PE, Brazil \\ ${ }^{3}$ Professor at the Department of Anatomy, Biosciences Center (CB), Federal University of Pernambuco (UFPE), Recife, PE, Brazil
}

Disclose and conflicts of interest: none to be declared by all authors

\section{ABSTRACT}

Introduction: the inferior vena cava is an important blood vessel to the venous drainage of the body's inferior portion and inferior members. During its development, it may occur some anatomic variations that are relevant from the clinical and surgical point of view, because of this fact, the professionals should have awareness of the possibilities of differences of normal anatomy.

Purpose: the main objective of this study was to describe an imaging finding of the inferior vena cava of a young patient and to correlate this anatomical variation with the clinical importance of their knowledge.

Case Report: young male patient, after an episode of digestive bleeding, recurrent diarrhea and occasional constipation for 2 months, looked for medical clinic service for investigation, being requested the accomplishment of ultrasonographic examinations and computed tomography of total abdomen. This work describes a case of duplication of the inferior vena cava, diagnosed after ultrasonography and computed tomography. We also carried out a study in the medical literature about the importance of the professional to know the anatomical variations of the inferior vena cava, especially in general and vascular surgical procedures, and his investigation through imaging tests. Ultrasound examination revealed the presence of a duplication of the infra-renal inferior vena cava, confirmed by computed tomography. Clinical evaluation and biochemical blood test results diagnosed the patient with lactose intolerance.

Conclusion: the knowledge of variations of the inferior vena cava is important to precise diagnostic from images and should be considered when planning abdominal surgeries and in endovascular procedures.

Key words: Anatomic variation; Radiography abdominal; Inferior vena cava; Vascular surgical procedures; Vascular malformations.

\section{Introduction}

The inferior vena cava (IVC) is one of the main veins in the organism, being an important retroperitoneal structure and must be observed in every imaging exam. ${ }^{1}$ Four segments (hepatic, adrenal, renal e infrarenal) constitute the IVC, which has its origin in the union of two common iliac veins around the fifth lumbar vertebra, assuming an upward direction, that passes to the right of the abdominal aorta by the surface of the psoas major muscle, flowing into the right atrium. To summarize, its function is to drain the blood from inferior members, a great part of the abdominal wall and back and, also, the organs from abdominal and pelvic areas. ${ }^{2-4}$

Embryologically, its development is quite complex, involving processes of growth, regression and anastomosis of some blood vessels during fetal period, mainly between the sixth and eighth weeks. It's shaped by three pairs of veins: posterior cardinal, subcardinal and supracardinal veins each one with its period of dominance between fourth, sixth and eighth weeks, respectively. ${ }^{1,5,6}$ Like this, there is the formation of a more established system, where the caudal portions of the posterior cardinal veins will give rise to the common iliac veins. The most cranial portion of the right subcardinal vein origins the hepatic portion of ICV, instead. This subcardinal vein also forms the suprarenal and renal portions of ICV, while the infrarenal portion of ICV is formed by the right supracardinal. It's worth emphasizing that the gonadal veins are originated from subcardinal veins and the left adrenal (suprarenal) is originated by left subcardinal vein. The superior segments of left and right supracardinal veins will be responsible for the formation of hemiazygos and azygos veins, respectively. ${ }^{5,7,8}$

This complex process of embryonic development of ICV may result in many anatomic variations ${ }^{9}$ that, that although are rare, can cause numerous functional 
alterations in the organism and so, are of great relevance from the clinical and surgical points of view. $^{10}$

The carries of alterations can be symptomatic or asymptomatic ${ }^{11}$ however, most part of these alterations are found accidentally. ${ }^{12}$ The lack of symptoms in some carriers of IVC interruption is justified by a great development in the azygos or hemiazygos vein, which is $0.6 \%$ of interruption cases. In the asymptomatic cases pain, edema and varicose veins in inferior members, pain and collateral circulation in the abdomen, hematochezia and varicocele are commonly observed. Besides that, it's usual the occurrence of deep venous thrombosis (DVT), thromboembolism and hepatic venous thrombosis, ${ }^{11}$ being the bilateral DVT present in $50 \%$ of the DVT cases in carries of IVC variations. ${ }^{9}$

This way, before the significant prevalence of this finding, it's clear that the knowledge of the IVC anatomic variations is of major importance in the systematization of surgical and radiologic anatomy of these blood vessels. Therefore, the objective of this work is to talk about a finding through images of inferior vena cava (IVC) anatomic variation in a young patient. Besides the study of this variation, some concepts related to anatomy, functionality and to the clinical importance of the inferior vena cava variations, as well as its repercussions in the surgical and radiological field, will also be reviewed.

\section{Case Report}

Young male patient, 18 years old, white, with an episode of digestive bleeding, recurrent diarrhea and occasional constipation for 2 months, looked for medical clinic service for investigation. The patient had already had appendectomy surgery when he was 9 years old, but without any previous disease. An ultrasound of the total abdomen was requested, in which the anatomic variation was verified: presence of a duplication in the infrarenal inferior vena cava (Fig. 1), with the left inferior vena cava draining to the renal homolateral vein. Other abdominopelvic organs where found without any signs of abnormality. To a better investigation, a computed tomography of the total abdomen (Fig. 2) was requested. In order to be less invasive with the patient, we chose not to order computed tomography (CT) with intravenous contrast medium. In addition, the availability of equipment, limited resources for diagnosis and clinical indication were considered. The IVC duplicity apparently did not cause any physiological alteration in the patient, who was diagnosed with lactose intolerance, the cause of the clinical condition reported before. It should be borne in mind that the duplication of the inferior vena cava may be related to other more extensive anatomical variations, related to the left renal vein, iliac veins, azygos, hemiazygos and thoracic and abdominal viscera.

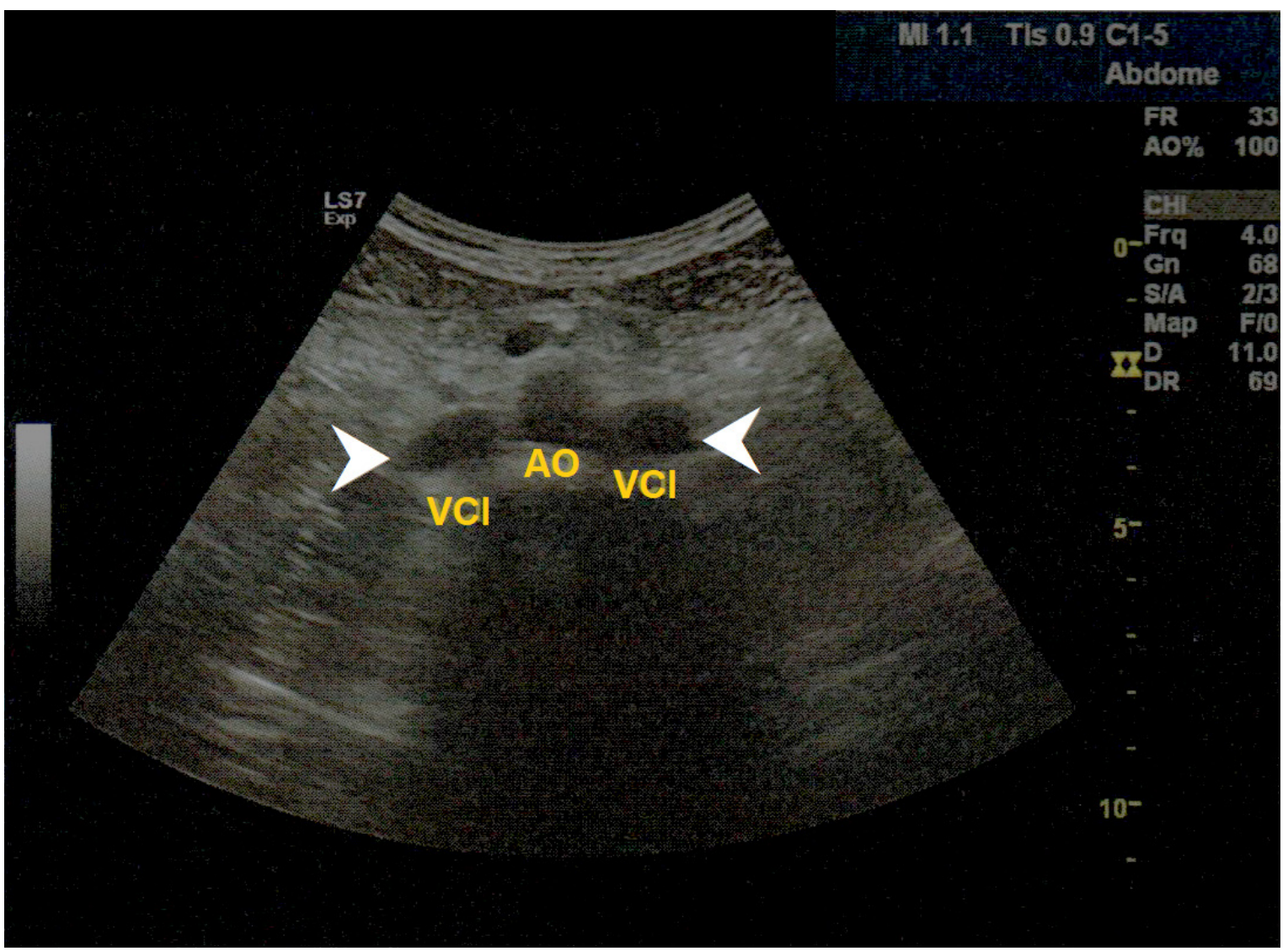

Figure 1. Ultrasound picture of the cross section of the inferior vena cava duplicity. Aorta artery (AO), left and right inferior vena cava (VCl, white arrows). 


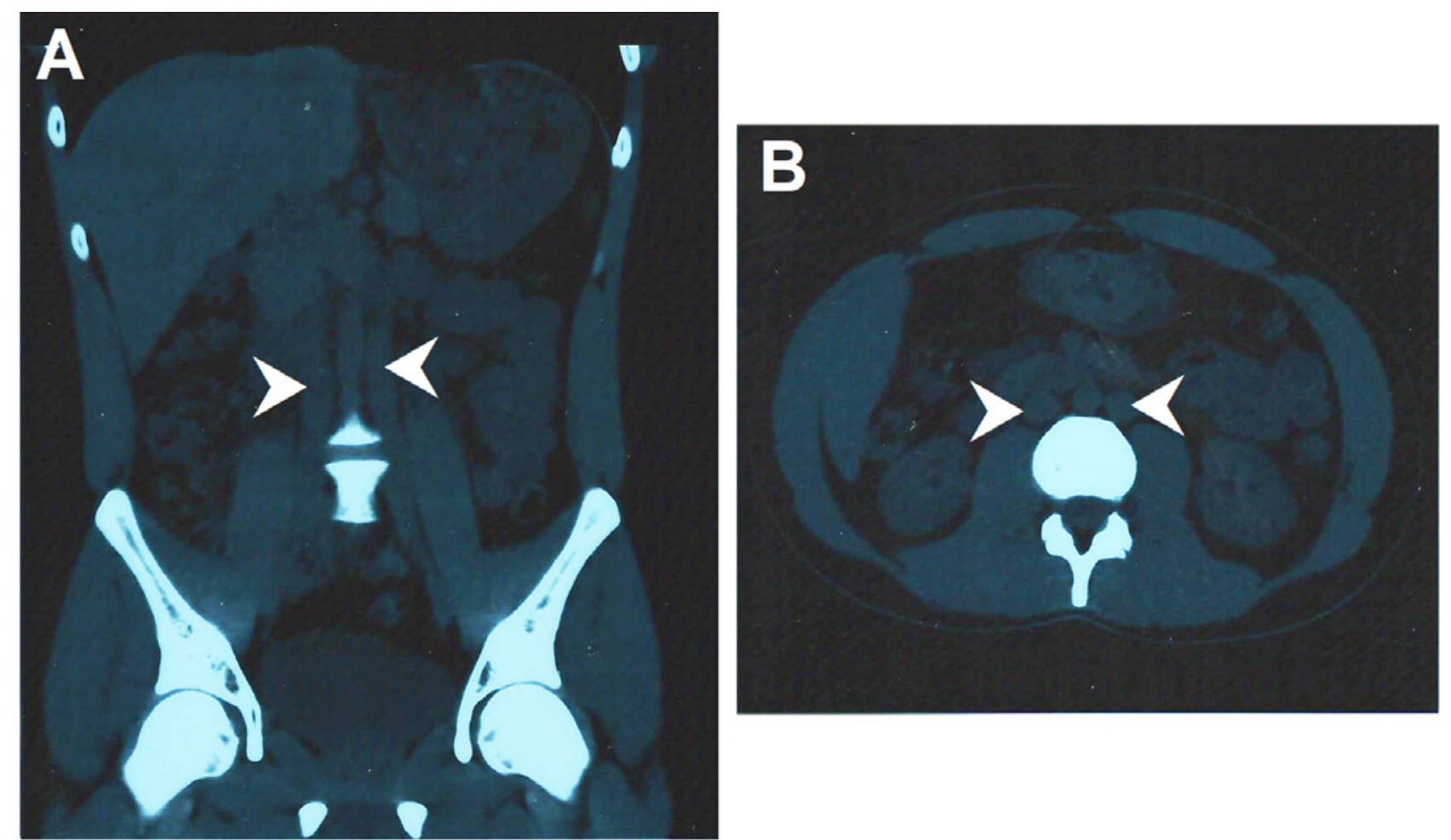

Figure 2. Computed tomography (CT) of the total abdomen. In A, coronal cut showing two inferior vena cava (white arrows). In B, axial cut also showing two inferior vena cava (white arrows) in both sides of aorta artery

\section{Discussion}

Anatomic variations of the inferior vena cava (ICV) are results of an abnormal embryo formation process and vascular development, and the duplication of the IVC is the main alteration. The most prevalent and more clinically important types of alterations are: left IVC ( 0.2 to $0.5 \%$ of the population), double IVC (1 to $3 \%$ of the population), circum-aortic left renal vein (1.5 to $8.7 \%$ of the population), retroaortic left renal vein, among others, such as subhepatic IVC agenesis, retrocaval ureter, IVC's transposition. ${ }^{6,9,12}$ Were also described retroureteral IVC, left IVC, retrocaval IVC, IVC with drainage through the azygos veins and IVC interruption with continuation by the azygos vein..$^{13}$ The variations of duplication of IVC correspond to about a third of these variations. ${ }^{11}$ Anderson et al. ${ }^{14}$ attributed the IVC duplicity to the persistence of both supracardinal veins.

However, the prevalence of said duplication is somewhat controversial. Most studies show the rareness of the cases, with an incidence of about 0.2$3 \%$ of normal individuals and reveal that the diagnosis usually occurs accidentally in radiological exams or in research for organic dysfunctions, by scanning in computerized tomography, magnetic resonance imaging or compression ultrasonography with color Doppler evaluation (US). ${ }^{9,11,15-19}$ According to Bass et $a l .{ }^{15}$ e Babu et al. ${ }^{20}$ the anomalies of IVC are rare and clinically asymptomatic, numerous times going unnoticed.

It's possible to find older studies that reveal a bigger incidence rate, approximately 1 in 374 normal individuals, revealing that the findings of double IVC aren't unusual. ${ }^{12}$ According to Baeshko et al. ${ }^{16}$ the congenital anomalies of IVC are more frequent in young males.

A literature review study, presented by Kapetanakis et al., ${ }^{12}$ showed that the data from populational prevalence rate, in other words, reported incidence, in Asian populations, mainly China, Japan and India, and those reported in Western Europe are very similar. According to Malaki et al., ${ }^{9}$ the duplicity of IVC were reported overall in men, corresponding to a percentage of $62 \%$, as well as the cases of aplasia/ agenesis. However, the IVCs interruptions were in bigger percentage in women.

In this way, a greater emphasis should be given to the clinical and especially surgical importance of a precise diagnosis, in the procedure of organ transplantation and retroperitoneal surgeries, ${ }^{21}$ such as radical nephrectomy, sympathectomy or ureteral surgery, aside from a special alert to possible thromboembolic events, ${ }^{9,12,22,23}$ The preoperative evaluation is notably important once these anatomic variations of IVC are noiseless, ${ }^{18,24,25}$ being diagnosed in routine dissection studies, retroperitoneal surgeries or by imaging exams, that were solicited with other purposes. ${ }^{26}$

The patient from the referred case did not report previous cardiovascular complaints, turning the venous anomaly to be diagnosed by finding through images. According to Artico and contributors ${ }^{1}$, the anatomic variations of this vase are a clinical finding 
relatively atypical during surgery or diagnostic procedures in patients without symptoms.

In some case reports were described heart defects associated with anomalous inferior vena cava. ${ }^{14,18}$ It could also be associated with other venous anomalies, observed in suprarenal and gonadal veins ${ }^{27}$ or in horseshoe kidney. ${ }^{28}$ Some other comorbidities can still be found such as splenic anomalies, intestinal malrotation, pulmonary dysgenesis, renal agenesis, dextrocardia or other congenital heart disease. ${ }^{29}$ Nevertheless, in the present description no other anatomic variation has been found.

The description of these anatomic variations has clinical importance because it is crucial to increase the safety of surgical procedures, of interventional radiology, of diagnostic imaging of retroperitoneal masses and for the treatment of thromboembolic disease. $^{30}$

The presence of duplicity or interruption of IVC is indicative of risk for deep venous thrombosis (DVT) in the inferior members, ${ }^{18,31}$ essentially in young patients (under 30 years old) and when the iliac veins are affected. It is important to consider that thrombi can extend to the additional IVC and not be detected or be subdiagnosed, requiring protection from both veins with vena cava filters. Occasionally, when there is recurrence of embolism after the placement of a filter it could be evidence of the presence of duplication, in this case it is suggested a venography of the femoral vein to diagnose the duplication. ${ }^{10,11,31}$

In the indications of IVC filter placement what may occur is the insufficiency of the venous network or the slowness / swirling of blood flows from the lower limbs and pelvis towards the heart, leading to thrombosis' development, especially in the iliac and femoral veins, even so it is more common in patients with IVC agenesis. ${ }^{29}$ In that way, according to Doe and Ryu, ${ }^{25}$ to the placement of the filter of IVC, the interventionist radiologist should take into consideration the possibility of existence of anatomic variations of this blood vessel, mainly because many of these anatomic considerations are clinically silent.

Another clinical relevance for this variation is the renin sampling for hypertensive patients, since the duplicity of the IVC may dilute the sample of the left renal vein. Because of that, cavography or other imaging techniques should be executed to avoid dilution, the catheter being as close as possible to the renal wire. ${ }^{1}$

In reality, few are the cases of double IVC with symptomatic patients. Even fewer are the conditions in which the indicated procedure is the placement of filters of vena cava. The pathophysiology of deep venous thrombosis (DVT) in double IVC patients is explained by the slow venous flow, despite the compensatory mechanism of collateral blood circulation, as an example, the distension of the venous return system azygos-hemiazygos, which could lead to venous hypotension of the extremities, predisposing to the formation of thrombi, typically bilateral, seen in 50\% of the cases ${ }^{32}$. The DVT is frequently the first symptom of a congenital anomaly of IVC. When asymptomatic, the IVC variations' treatment is conservative for most patients. ${ }^{16}$

When related to the diagnosis of IVC duplicity, it's extremely hard to be done by older radiological methods, the CT imaging being necessary, as well as any other retroperitoneal structures, especially when in the presence of large amounts of retroperitoneal fat. Through CT, the IVC can easily be mapped since its origin, with the technique being non-invasive and effective to the diagnosis of diseases of the retroperitoneal space, particularly to detect anomalies in the main blood vessels, such as the aorta artery or IVC, in asymptomatic patients. ${ }^{1}$ Nowadays, the multidetector computerized tomography (MDCT) allows data acquisition from volumetric image of high spatial resolution of two-dimension (2D) and threedimension (3D) after the processing, which allows the complex vascular malformations visualization in an understandable way. ${ }^{4}$

Nevertheless, this diagnosis can be erroneous in paraaortic adenopathy cases, essentially because of the lymphomatous or metastatic disease, in which the lymph nodes get bigger and, therefore, making it possible to visualize single masses or conglomerate bodies surrounding the aorta or the IVC. This anatomic variation may be mistaken for lymphadenopathy or with a retroperitoneal mass, ${ }^{20,33,34}$ making it necessary to know the different IVC anatomic variations so that the radiologists may avoid wrong interpretations. ${ }^{4,20}$ According to Baeshko et al. ${ }^{16}$ the differentiation in the diagnosis of IVC anomalies is important in the choice of a treatment method and additional prognosis.

The investigation of IVC variations through imaging exams is even more relevant to the investigation of DVT cases recurring in young individuals, principally if the thrombosis is bilateral ${ }^{11}$. Besides that, the knowledge of IVC anatomy in the preoperative is capable of avoiding numerous surgical complications in radical nephrectomies, sympathectomy, retroperitoneal surgeries, organ transplants, or ureteral surgery. ${ }^{9,12}$ Another surgical relevance of the IVC variations is referred to the placement of the filters in this blood vessel in patients who carry DVT. The acknowledgment of the many variations allows a better surgical planning and avoid recurring events of post-surgical pulmonary embolism in cases of double vena cava ${ }^{9}$. In that way, the new radiological modalities emerge with great importance in this area, for allowing a better visualization of the anatomic relations, being necessary basic knowledge of embryology and the anatomic variations of the IVC to a correct interpretation and description by the radiologist, with special attention to the alterations that may result in clinical and surgical implications. 
The anatomic variation of IVC may lead to surgical complications in procedures in the retroperitoneum, and because of that it is important that the surgeon is familiar with these, so that he can anticipate and successfully conduct some of the potential problems. The variations of venous structures tend to be dilated and tortuous, making the injury risk more possible. Therefore, the lack of preoperative knowledge of retroperitoneal venous variations, especially with an unexpected presence of IVC in a unusual position, could have disastrous consequences, such as the risk of bleeding during the removal of organs or an intraoperative trauma of retroperitoneal surgery, putting at risk the life of the patient, being a challenge to the surgical professional. $5,8,23,36,37$ In nephrectomy cases, whose donor has the duplication of the IVC, it has been reported postoperative complications, like scrotal edema. ${ }^{38}$

Variations of the arterial and venous system of the abdomen and pelvis have important clinical in abdominal surgeries (of the gastrointestinal tract, kidneys and endopelvic organs), in abdominal laparoscopic procedures and radiological intervention. ${ }^{39}$ Congenital anomalies of IVC are rare, however, it is of great relevance the knowledge from the carrier (patient), radiologists, surgeons, general practitioners, urologists and oncologists of these variations, especially in organ donations cases. ${ }^{36,39,40}$ In renal transplant cases, the left renal vein may be shorter, apart from having urological anatomic variations and vascular ones that accompany them. ${ }^{7,8}$

Thus, the clinical importance of duplicity of the inferior vena cava in retroperitoneal surgeries, abdominal organ transplants, total nephrectomy, inferior vena cava ligation, and placement of vena cava, especially in cases of thromboembolic disease, can be clearly demonstrated. pharmacological therapy with anticoagulants and radiological abdominal intervention. ${ }^{39}$ In this sense, the real necessity of all transplant surgeons to confirm the anatomy of all blood vessels involved in the surgical procedure stands out, so that if any anomaly is present, they should be prepared to handle these situations.

According to Arudchelvam et al. ${ }^{41}$ the lack of knowledge in this rare anatomic variation may result in serious mistakes in diagnosis, surgical and therapeutic procedures. However, it can be easily avoided by its recognition in magnetic resonance and computed tomography. Besides that, according to Bubb et al., ${ }^{42}$ there are still big anatomic variations regarding the presence and distribution of venous valves and regarding the location of the ostia of the inferior vena cava venous tributaries, that together with other possibilities of alteration in normality, should be considered in surgical procedures.

The lymphatic drainage may be different in patients with venous variations. Extensive dissection of lymph nodes, for an example, is recommended for patients with testicular tumors and duplication of the IVC, ${ }^{8}$ ratifying the conduct of verification of normal anatomy and all the other structures implied in the surgical field and other various pathologies.

According to Freire Filho et al. ${ }^{43}$ the use of abdominal CT without contrast, when pertinent, can be included later in the contracted phase, guaranteeing the full potential of the method. To Barranhas et al., ${ }^{44}$ the conventional CT, even without contrast, is important to prevent any type of iatrogenic in a future procedure. In addition, in most clinical situations, practitioners do not have easy access to contrast imaging, multiplanar $3 \mathrm{D}$ reconstruction, or other imaging techniques.

\section{Final Considerations}

The knowledge of anatomic variations of the inferior vena cava (IVC) is important to precise imaging diagnosis, to avoid diagnostic errors such as tumors or retroperitoneal lymphadenopathy, possible vascular compressions and association with deep venous thrombosis, but also should be considered when planning abdominal surgeries and endovascular interventions, over all to professionals that are specialized in images and surgeons, to avoid possible complications. In this way, the IVC duplicity is an unusual finding, but clinically significant.

\section{References}

1. Artico M, Lorenzini D, Mancini P Gobbi P, Carloia S, David V. Radiological evidence of anatomical variation of the inferior vena cava: Report of two cases. Surg Radiol Anat 2004;26:153-156.

2. Alves EC, Ferro GBR, França LKL, Jacó MB, Pitta GBB. Ausência de veia cava inferior: relato de caso. J Vasc Bras 2010;9:254-256.

3. Espírito Santo TM, Bacalhau F, Paschôa AF, Raci D (2015) Anatomia tomográfica da veia cava e das veias renais: aspectos relevantes para implante de filtro de veia cava. J Vasc Bras 14:10-15. https:/ / doi. org/10.1590/1677-5449.20130106.

4. Coco D, Cecchini S, Leanza S, Viola M, Ricci S, Campagnacci R. Inferior Vena Cava Duplication: Incidental Case in a Young Woman. Case Reports in Radiology 2016:1-4.

5. Eldefrawy A, Arianayagam M, Kanagarajah P, Acosta K, Manoharan M. Anomalies of the inferior vena cava and renal veins and implications for renal surgery. Cent European J Urol 2011;64:4-8.
6. Yang C, Trad HS, Mendonça SM, Trad CS. Congenital inferior vena cava anomalies: a review of findings at multidetector computed tomography and magnetic resonance imaging. Radiol Bras 2013;46:227-233.

7. Kellman GM, Alpern MB, Sandler MA, Craig BM. Computed tomography of vena caval anomalies with embryologic correlation. Radiographics 1998;8:533-556.

8. Chen H, Emura S, Nagasaki S, Kubo KY. Double inferior vena cava with interiliac vein: a case report and literature review. Okajimas Folia Anat Jpn 2012;88:147-151.

9. Malaki M, Willis AP, Jones RG. Congenital anomalies of the inferior vena cava. Clin Radiol 2012;67(2):165-171.

10. Patel S, Cheema A, Karawadia T, Carson M. Management of acute lower extremity deep venous thrombosis in a patient with duplicated inferior vena cava and contraindication to anticoagulation: case 
and review of the literature. BMJ Case Rep 2018;4;2018.

11. Koc Z, Oguzkurt L. Interruption or congenital stenosis of the inferior vena cava: prevalence, imaging, and clinical findings. Eur J Radiol 2007;62(2):257-266.

12. Kapetanakis S, Papadopoulos C, Galani P, Dimitrakopoulou $G$, Fiska A. Anomalies of the inferior vena cava: a report of two cases and a short review of the literature. Folia Morphol (Warsz) 2010;69:123-127.

13. Xue HG, Yang CY, Asakawa M, Tanuma K, Ozawa H; Duplication of the inferior vena cava associated with other variations. Anat Sci Int 2006;82(2):121-125.

14. Anderson RC, Adams PJr, Burke B (1961) Anomalous inferior vena cava with azygos continuation (infrahepatic interruption of the vena cava). J Pediatr 59(3):370-383.

15. Bass JE, Redwine MD, Kramer LA, Huynh PT, Harris JH Jr. Spectrum of congenital anomalies of the inferior vena cava: cross-sectional imaging findings. Radiographics 2000;20(3):639-652.

16. Baeshko AA, Zhuk HV, Ulezko EA, Goresckaya IV, Oganova EG, Dudarev VS, Orlovski YN. Congenital Anomalies of the Inferior Vena Cava and their Clinical Manifestation. EJVES Extra 2007;14(1):8-13 17. Masood J, Barua JM. Duplication of the inferior vena cava. N Engl J Med 2007;356:e17.

18. Ertuğrul I, Doğan V, Örün UA, Karademir S. A rare association: inferior vena cava anomalies and congenital heart diseases. Turk Kardiyol Dern Ars 2015;43(8):717-719.

19. Valavan SS, Castellanos B, Rich S, Goldberg R (2017). Double inferior vena cava with variant hemiazygos vein - a case report. IJAE 122(2):121-126.

20. Babu CSR, Lalwani R, Kumar I. Right Double Inferior Vena Cava (IVC) with Preaortic Iliac Confluence - Case Report and Review of Literature. J Clin Diagn Res 2014;8(2):130-132.

21. Babaian RJ, Johnson DE. Major venous anomalies complicating retroperitoneal surgery. South Med J 1979;72(10):1254-1258.

22. Shaw MB, Cutress M, Papavassiliou V, White S, Thompson M, Sayers R. Duplicated inferior vena cava and crossed renal ectopia with abdominal aortic aneurysm: preoperative anatomic studies facilitate surgery. Clin Anat 2003;16:355-357.

23. Mao YQ, Zhu SX, Zhang W. The iatrogenic injury of double vena cava due to misdiagnosis during the radical nephroureterectomy and cystectomy. World J Surg Oncol 2015;13:41.

24. Ichikawa T, Kawada S, Yamashita T, Niwa T, lino M, Koizumi J, Kawaguchi Y, Imai Y (2014). A case of right double inferior vena cava with circumcaval ureter. Jpn J Radiol 32(7):421-424.

25. Doe C, Ryu RK. Anatomic and Technical Considerations: Inferior Vena Cava Filter Placement. Semin Intervent Radiol 2016;33(2):88-92 26. Ureyen I, Kestel Z, Sahin EG, Karalok A, Turan T, Boran N, Tulunay G. Double vena cava inferior: A report of three cases. Asian Pac J Reprod 2013;2(3):248-250.

27. Sürücü HS, Erbil KM, Tastan C, Yener N. Anomalous veins of the retroperitoneum: clinical considerations. Surg Radiol Anat 2002;23(6):443-445.

28. Ichikawa T, Kawada S, Koizumi J, Endo J, Itou C, Matsuura K, Terachi T, Imai Y. Anomalous inferior vena cava associated with horseshoe kidney on multidetector computed tomography. Clin
Imaging 2013;37(5):889-894.

29. Gensas CS, Pires LM, Kruse ML, Leiria TLL, Gomes DG, Lima GG. Agenesis of the Inferior Vena Cava. Rev Bras Cardiol Invasiva 2012;20:427-430.

30. Castro-Iglesias AM, Díaz-Bermúdez J, Gargo-Ferreiro C, NoyaCastro A. Double vena cava inferior. Actas Urol Esp 2010;34(9):815826.

31. Pozzi A, El Lakis MA, Chamieh J, Carbonell BB, Villa F. The Typical Presentation Spectrum of Deep Vein Thrombosis Associated with Inferior Vena Cava Malformations. Thrombosis 2016;2016:1-5.

32. Prandoni $\mathrm{P}$, Lensing AWA, Cogo A, Cuppini S, Villalta S, Carta $M$, Cattelan AM, Polistena P, Bernardi E, Prins MH. The long-term clinical course of acute deep venous thrombosis. Ann Intern Med 1996;125(1):1-7.

33. Tisnado J, Amendola MA, Vines FS, Beachley MC. Computed Tomography of a Double Inferior Vena Cava: the "Double Cava" Sign. Computed Tomography 1979;3(3):195-199.

34. Malgor RD, Sobreira ML, Boaventura PN, Moura R, Yoshida WB. Filter placement in duplicated inferior vena cava: case report and review of the literature. J Vasc Bras 2008;7(2):167-170

35. Petik P. Inferior vena cava anomalies and variations: imaging and rare clinical findings. Insights Imaging 2015;6(6):631-639.

36. Polguj M, Szubert W, Topol M, Stefanczyk L. An unusual duplication of the inferior vena cava in a patient with endovascular repair for abdominal aortic aneurysm. Rom J Morphol Embryol 2015;56(2 suppl):875-878.

37. Kim M-H, Jun K-W, Moon I-S, Kim J-I. Clinical importance of congenital anomalies of the inferior vena cava in organ procurement surgery from a deceased donor: two case reports. Ann Surg Treat Res 2016;91(5):260-264.

38. Christakis PG, Cimsit B, Kulkarni S. Complication Arising from a Duplicated Inferior Vena Cava following Laparoscopic Living Donor Nephrectomy: A Case Report. Transplant Proc 2012;44(5):1450-1452. 39. Chentanez V, Nateniyom N, Huanmanop T, Agthong S. Coexistence of the double inferior vena cava with complex interiliac venous communication and aberrant common hepatic artery arising from superior mesenteric artery: a case report. Folia Morphol (Warsz) 2018;77(1):151-155.

40. Gandhi SP, Modi P, Sutariya H, Patel K. Rare Anatomical Variation of Dual IVC with Left Sided IVC Draining into Hemiazygous Vein - A Case Report. J Clin Diagn Res 2016;10(3):TD14-TD15.

41. Arudchelvam JD, Gunawardena RMTM, Dissanayake LR, Cassim MR, Wijeyaratne SM. Double inferior vena cava: beware! Ceylon Med J 2013;58:38-39.

42. Bubb K, du Plessis M, Hage R, Tubbs RS, Loukas M. The internal anatomy of the inferior vena cava with specific emphasis on the entrance of the renal, gonadal and lumbar veins. Surg Radiol Anat 2016;38:107-114.

43. Freire Filho EO, Jesus PEM, D'Ippolito G, Szejnfeld J. Tomografia computadorizada sem contraste intravenoso no abdome agudo: quando e por que usar. Radiol Bras 2006;39(1):51-62

44. Barranhas AD, Ney MSCJ, Alves M, Santos AASMD, Nacif MS. Which is your diagnosis? Radiol Bras 2013;46(2):XIII-XV.
Corresponding author

Jaciel Benedito de Oliveira

jaciel.oliveira@ufpe.br 